Submitted to the: 2003 Particle Accelerator Conference

Portland, Oregon, May 12-16, 2003

\title{
MULTI-MILLION-TURN BEAM POSITION MONITORS FOR RHIC
}

\author{
T. Satogata *, P. Cameron, P. Cerniglia, J. Cupolo, C. Dawson, C. Degen, J. Mead, K. Vetter- \\ Brookhaven National Laboratory, Upton, NY 11973-5000, USA
}

\section{Abstract}

During the RHIC 2003 run, two beam position monitors (BPMs) in each transverse plane in the RHIC blue ring were upgraded with high-capacity mezzanine cards. This upgrade provided these planes with the capability to digitize up to 128 million consecutive turns of RHIC beam, or almost 30 minutes of continuous beam centroid phase space evolution for a single RHIC bunch. This paper describes necessary hardware and software changes and initial system performance. We discuss early uses and results for diagnosis of coherent beam oscillations, turn-by-turn (TBT) acquisition through a RHIC acceleration ramp, and ac-dipole nonlinear dynamics studies.

\section{INTRODUCTION AND ARCHITECTURE}

The RHIC BPM system[1, 2, 3] consists of $16023-\mathrm{cm}$ cryogenically-stable striplines per plane per ring: 72 dualplane BPMs are distributed through the interaction regiosn (IRs), and 176 single-plane BPMs are located at each arc $\beta_{\text {max }}$. Signals are cabled through $6 \mathrm{~dB}$ reflection attenuators and $20 \mathrm{MHz}$ lowpass filters to analog/digital integrated front ends (IFEs). Each IFE contains independent electronics boards for two measurement planes, including active 20 and $40 \mathrm{~dB}$ gain stages, 16-bit digitizers for $1 \mu$ mresolution over a $\pm 32 \mathrm{~mm}$ measurement range, and Motorola 56301 fixed-point digital signal processors (DSPs) for data reduction and acquisition control. Are IFEs are in the tunnel, $2 \mathrm{~m}$ above the cryostat, or in tunnel alcoves for radiation protection; IR IFEs are located in equipment buildings.

During data acquisition, each IFE calculates the trigger and digitizer status, digitized raw signals, and beam position once per turn for a single RHIC bunch. Upon receipt of a beam-synchronous trigger, all RHIC BPMs can digitize up to 1024 consecutive turns and stream them to the local DSP buffer. This data is then passed along an IEEE 1394 connection to VME memory and the RHIC control system. This upgrade extends the on-board memory space of selected RHIC BPMs to provide much longer buffers for purposes of beam studies, energy-ramp beam instability study, and analysis of well-established slow coherent oscillations of RHIC beams. With average orbit data available only as fast as $1 / 2 \mathrm{~Hz}$ and 1024-turn TBT acquisition covering only $13 \mathrm{~ms}$, this upgrade provides TBT data capability that easily spans the BPM system's former frequency-domain blind spot of $1-100 \mathrm{~Hz}$.

* Work performed under Contract Number DE-AC02-98CH10886 with the auspicies of the US Department of Energy; author email satogata@bnl -gov

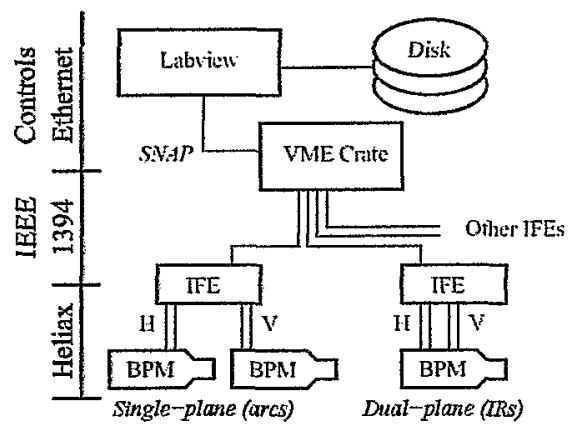

Figure 1: Architecture of the BPM million-turn upgrade

\section{UPGRADE CHANGES}

\section{Hardware}

The existing RHIC BPM digital electronics has a PCI mezzanine carrier (PMC) slot available, which made the hardware upgrade straightforward. The Motorola 56301 DSP has a built-in PCI interface, allowing a glueless interface to the PMC memory card. A commercially available PMC memory Card from RAMIX (model 551) was purchased and installed. This memory card provides 512 Mbytes of SDRAM, which is used to buffer the TBT data until readout. A special million-turn event was defined on the global event link to provide a start trigger for data acquisition; once data acquisition is started, the DSP ignores interrupts until the full acquisition is complete and data is read out.

The RAMIX 551 PMC memory card consumes $600 \mathrm{~mA}$ at $3.3 \mathrm{~V}$. The existing voltage regulator on the BPM electronics is an On SemiconductorCS5203A-3GT3 LDO. The power dissipated from this regulator increased by $(5.0 \mathrm{~V}$ $3.3 \mathrm{~V}) \times 600 \mathrm{~mA}=1 \mathrm{~W}$, requiring an additional heat sink.

Four BPM planes with IFEs located in the 2 o'clock instrumentation building were modified in late April 2003, during RHIC polarized proton run. In the RHIC sitewide nomenclature, they are bo2-bh8 and bhl0 (horizontal) and bo2-bv7 and bv9 (vertical). Typical phase advances between these BPMs in all RHIC optics are 70 to 80 degrees, providing orthogonality for phase space reconstruction.

\section{Software}

The DSP software was modified to add a new special million-turn mode of operation, preserving the full base functionality (average orbit and 1024-turn TBT acquisition) of the upgraded BPMs. Each TBT data point includes raw digitizer values, calculated beam position, and a status word, requiring 4 words of storage per turn. This allows 


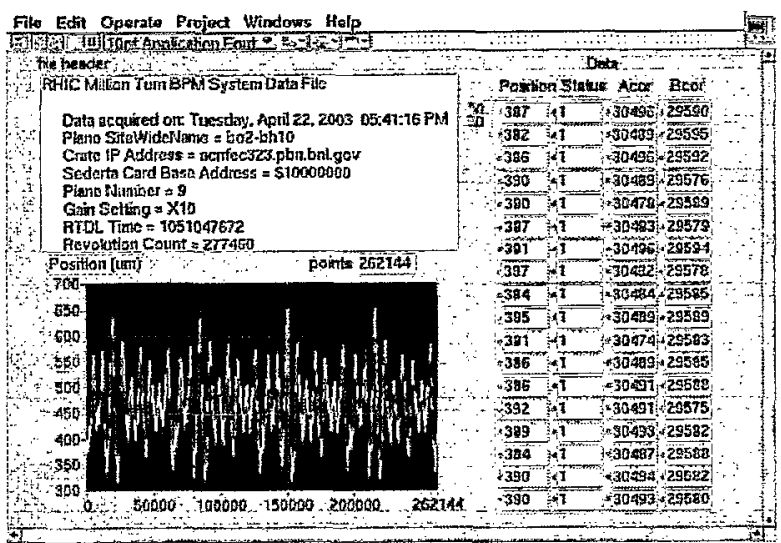

Figure 2: The Labview interface for a single RHIC millionturn plane, showing a supposed 256 kturns of acquisition and clear coherent beam motion in storage conditions. A handshaking bug results in report of the same 64 kturns four times. BPM TBT RMS noise with good signal conditions and $\times 10$ active gain is $5-10 \mu \mathrm{m}$.

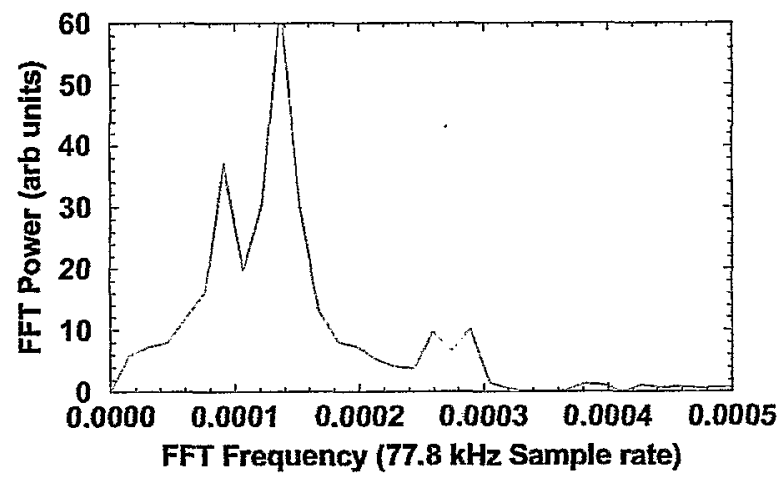

Figure 3: FFT of coherent oscillations from data of Fig. 2, showing clear peak at $10.66 \mathrm{~Hz}$ and $7.16 \mathrm{~Hz}$. The RHIC revolution frequency is $78.8 \mathrm{kHz}$.

recording of up to 128 million consecutive turns of RHIC beam, or over 28 minutes of beam phase space evolution at the $\mathrm{RHIC}$ revolution rate of $78 \mathrm{kHz}$.

After the readout has completed, the data is sent over a 1394 serial bus link to a Sederta VME-1394 bridge. With the existing infrastructure, the Sederta card has only 1 Mbyte of memory assigned for each plane. To maintain hardware and software compatibility, a handshake protocol was developed to transfer the information from the BPM electronics and controls front-end computer to a unix platform running Labview in $512 \mathrm{kbyte}$ chunks using a simple network communications protocol called SNAP. At present there are handshaking problems leading to repeated retrieval of the same 512 kbyte buffer, so meaningful data acquisition is limited to 64 kturns. (See Fig. 2.)

The handshake protocol makes the data transfer time very long, and it can take a few hours to read out the full 128 million turns for a single plane. Upgrades will con- tinue to optimize this readout, including the use of a separate VME memory card to eliminate the DSP to controls handshake.

\section{EXPERIENCE AND APPLICATIONS}

The first application of the RFIIC million-turn system was immediately apparent with the first good data acquisition. 256 kturns of data were acquired at storage energy to show clear signs of $10.66 \mathrm{~Hz}$ and $7.16 \mathrm{~Hz}$ beam oscillations, as shown in Figs. 2 and 3. These oscillations are the dominant source of coherent beam motion in low-beta storage conditions. No coherent oscillation is seen in the vertical plane.

This mechanical frequency has also been observed in horizontal vibrations of RHIC triplet magnets [4], though at slightly different frequencies of $7.75 \mathrm{~Hz}$ to $11 \mathrm{~Hz}$ as measured in the low-beta triplets at the 6 and 8 o'clock locations. With phase space reconstruction, the location of the source can be narrowed within the ring, and further tests will be performed in the cryogenic and main control rooms to investigate effects of changing recirculator conditions on this oscillation.

Acquisition of a single plane at injection as seen in Fig. 4 showed much different coherent beam motion, with clear oscillations seen near $30 \mathrm{~Hz}$ and $60 \mathrm{~Hz}$. The root cause of this signal is presumed to be main dipole ripple in the arcs at low energy, though it is under investigation.

The limited 1024-turn buffer size of the RHIC BPM system hampered their use during RHIC ac dipole optics and nonlinear dynamics studies[5], since the ac dipole excitation ramp time is several hundred milliseconds to adia-
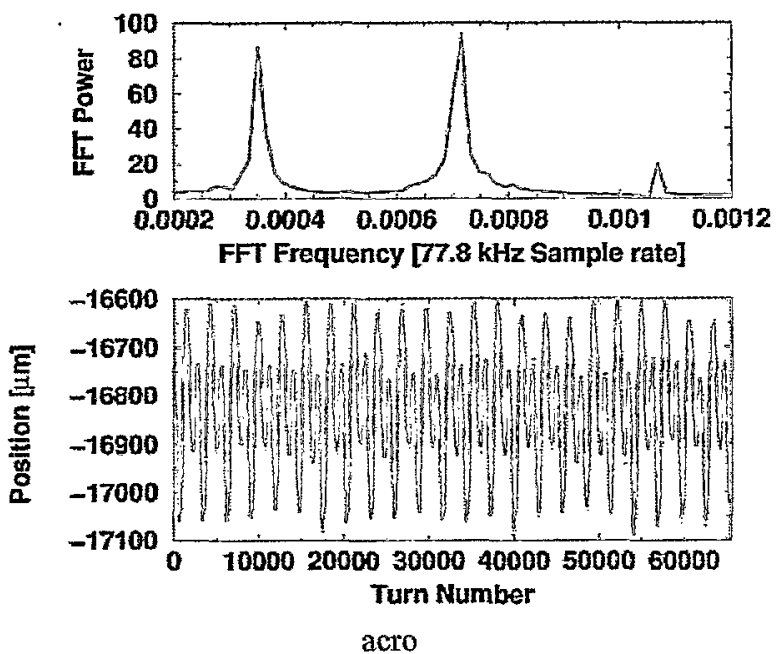

Figure 4: The Labview interface for a single RHIC millionturn plane, showing a supposed 256 kturns of acquisition and clear coherent beam motion in storage conditions. A handshaking bug tesults in report of the same 64 kturns four times. BPM TBT RMS noise with good signal conditions and $\times 10$ active gain is $5-10 \mu \mathrm{m}$. 
batically excite coherent oscillations and avoid emittance blowup. The million-turn BPMs will enable acquisition of TBT data and full phase space reconstruction during a full cycle of ac dipole ramp-up, flattop, and ramp-down. This permits fine-tuning and of the ac dipole excitation, as well as explorations of phase space smear and other topics in nonlinear beam dynamics[6].

When the full system is commissioned, a provocative planned use is the acquisition of an entire RHIC acceleration and squeeze ramp. These ramps typically take 5-10 minutes, easily within the acquisition time of the millionturn BPM system. With tunemeter kicks occurring every two seconds, acquisition of all million-turn BPM planes will provide full beam centroid phase space on the acceleration ramp. This system will also permit detailed study of intensity-dependent transverse instabilities, particularly through transition energy, as observed and corrected during the 2003 RHIC deuteron-gold run[7,8].

\section{REFERENCES}

[1] T.J. Shea and R.L. Witkover, "RHIC Instrumentation", Beam Instrumentation Workshop 1998 (Stanford).

[2] T. Satogata et al., "RHIC Instrumentation", Nucl Instr Meth in Phys Res A 499 (2003) pp. 372-387.

[3] T. Satogata et al., "RHIC BPM System Performance, Upgrades, and Tools", EPAC2002 (Paris, France).

[4] C. Montag et al., "Measurements of Mechanical Triplet Vibrations in RHIC", EPAC2002 (Paris, France).

[5] M. Bai et al., "Measurement of Betatron Functions and Phase Advances in RHIC with AC Dipoles", WPAB072, these proceedings (May 2003).

[6] S. Peggs, "Nonlinear Diagnostics Using AC Dipoles", PAC 1999 (New York, New York).

[7] T. Satogata et al., "Commissioning of RHIC Deuteron-Gold Collisions", TPPB043, these proceedings (May 2003).

[8] M. Blaskiewicz et al., "Transverse Instabilities in RHIC", RPPB007, these proceedings (May 2003). 